\title{
Orthogonal and Non-orthogonal Cooperative protocols in WLANs
}

\author{
Jun Chen ${ }^{12}$, Karim Djouani ${ }^{2}{ }^{3}$ and Kamel Barkaoui ${ }^{1}$ \\ ${ }^{1}$ CEDRIC-CNAM Paris, France \\ ${ }^{2}$ LISSI Lab., University Paris-Est, Paris, France \\ ${ }^{3}$ F'SATIE/TUT, Pretoria, South Africa \\ chen_ju@auditeur.cnam.fr, djouani@univ-paris12.fr, barkaoui@cnam.fr
}

\begin{abstract}
The two main cooperative protocols in WLANs are the orthogonal and non-orthogonal ones. We consider this two different cooperative protocols with the amplify-andforward (AF) and decode-and-forward (DF) strategies. In each case, we study the information theoretic performance. Simulation results show that the non-orthogonal cooperative protocol achieves an optimal performance than the orthogonal one. Furthermore, using space-time coding in non-orthogonal protocols provides the additional coding diversity gains in the transmission.
\end{abstract}

Keywords: cooperation, orthogonal protocol, non-orthogonal protocol, space-time coding

\section{INTRODUCTION}

Wireless communication always suffers from multi-path fading and co-channel interference. Recently, cooperative diversity technique is generated by transmitting different versions of the signal via a relay set which create a virtual antenna array between the source and the destination. With the help of cooperation protocols, it can exploit the diversity as a multiple-in multiple-out (MIMO) system [2]. The three main strategies of cooperative communication are: amplify-andforward (AF), decode-and-forward (DF) and compress-and-forward (CF). In this paper, we focus on $\mathrm{AF}$ and DF strategies.

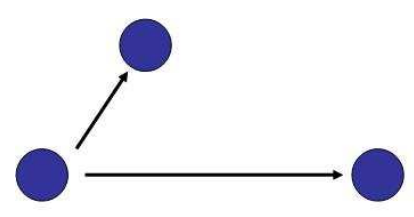

First time-slot

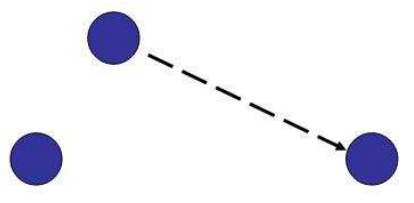

Second time-slot

FIGURE 1: Single-Relay Orthogonal Cooperative Protocol

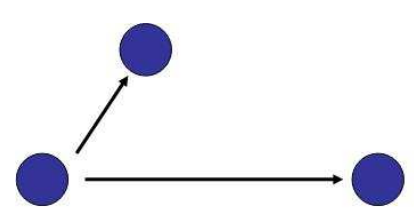

First time-slot

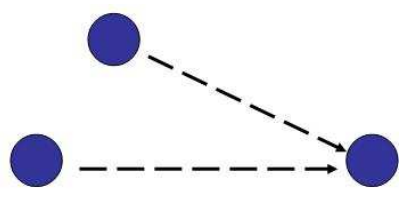

Second time-slot

FIGURE 2: Single-Relay Non-orthogonal Cooperative Protocol

Without loss of generality, a cooperation protocol composed of two time slots: During the first time slot, the source communicates with the relays and destination. In the second time slot, as shown in FIGURE 1, when only the relays communicate with the destination, the protocol is considered 
as orthogonal. On the contrary, as shown in FIGURE 2, the protocol is not orthogonal when both the source and the relays communicate with the destination.

Most prior works focused on AF strategy because of the simplicity. The relays only amplify and retransmit the signal received from the source (the signal received at the relay is distorted by fading and additive noise) and no demodulation or decoding is performed at relays in this case. The DF strategy offers good performance but have a higher complexity compared to the AF strategy in practice.

Laneman and Wornell first proposed AF strategy as a cooperative signaling scheme in [8]. They compute the bit error rate (BER) for uncoded AF strategy, and show that, despite the noise propagation from the partner, AF strategy performs significantly better than non-cooperative transmission. Laneman, Wornell and Tse [9][10] extend this work by deriving the outage probability which is assumed if a user does not successfully detect the partners symbols for AF strategy and DF strategy in quasi-static Rayleigh fading channel. It is shown that a simple point-to-point source-relay-destination protocol achieves a first-order diversity, the same as noncooperative transmission, and performs worse than non-cooperative transmission for a wide range of conditions. This is due to the relay may often transmit erroneous estimates of the symbols from the source.

In our work we studied orthogonal and non-orthogonal protocols with AF and DF strategies and give the best protocol choice. The remainder of the paper is organized as follows. We present the system model in section 2 . Section 3 gives the information theoretic performance analysis of the two cooperative protocols with both $\mathrm{AF}$ and DF strategies. The space-time coding design for non-orthogonal protocol is presented in section 4 . We evaluate the performance of our solution using the simulations in Section 5. Conclusions on this study are given in the last section.

\section{SYSTEM MODEL}

We consider in this work a wireless network like FIGURE 3 consists of one source $(s)$, one destination $(d)$ and one relay $(r)$. All the terminals are equipped with single antenna transmitters and receivers. Throughout this paper we assume that our research work lays on half duplex communication model, where relay will not receive and transmit same time and same frequency band. The channel between terminals are mutually independent and subject to quasi-static flat Rayleigh fading. Channel conditions between the terminals are known to the destination terminal.

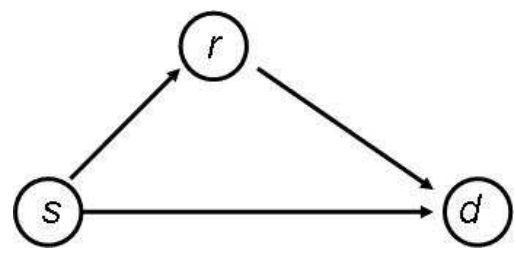

FIGURE 3: Single-Relay Cooperative Transmission Model

\subsection{Non-Orthogonal protocol with AF strategy (NAF)}

The signals received by the destination and relay in the first time slot can be defined as

$$
y_{s d}=w_{s d} h_{s d} x_{1}+n_{s d}
$$

and

$$
y_{s r}=w_{s r} h_{s r} x_{1}+n_{s r}
$$


respectively, where $w_{s d}^{2}$ and $w_{s r}^{2}$ are the average signal energies received by destination over channel $s \rightarrow d$ and $s \rightarrow r$, respectively. $h_{s d}$ and $h_{s r}$ are the random, complex-valued and unitpower channel gains between $s \rightarrow d$ and $s \rightarrow r . n_{s d} \sim \mathcal{C N}\left(0, N_{s d}\right), n_{s r} \sim \mathcal{C N}\left(0, N_{s r}\right)$ is the additive noises, and in general $w_{s d}^{2} \neq w_{s r}^{2}$.

The energy of received signal is given by

$$
E\left(\left|y_{s r}\right|^{2}\right)=E\left(\left|w_{s r} h_{s r} x_{1}\right|^{2}\right)+E\left(\left|n_{s r}\right|^{2}\right)=w_{s r}^{2} h_{s r}^{2}+N_{s r}
$$

In order to retransmit the signal with the same power as the sender did, the normalization gain $\beta$ for the amplification is

$$
\beta=\sqrt{\frac{1}{w_{s r}^{2} h_{s r}^{2}+N_{s r}}}
$$

Then, the destination receives a superposition of relay and source during the second time slot:

$$
y_{2}^{N A F}=w_{s d} h_{s d} x_{2}+v_{r d} h_{r d} \beta y_{s r}+n_{r d}
$$

where $v_{r d}^{2}$ is the average signal energy received at the destination through channel $r \rightarrow d$, the definition of $h_{r d}$ and $n_{r d}$ are the similar to $h_{s r}$ and $n_{s r}$.

So the equation (5) can be rewritten as:

$$
y_{2}^{N A F}=w_{s d} h_{s d} x_{2}+v_{r d} w_{s r} \beta h_{s r} h_{r d} x_{1}+\tilde{n}
$$

where $\tilde{n} \sim \mathcal{C N}\left(0, N_{0}\right)$ with $N_{0}=v_{r d}^{2} h_{r d}^{2} \beta^{2} N_{s r}+N_{r d}$.

As the summary, the transmission function is

$$
\mathbf{y}^{\mathrm{NAF}}=\mathbf{H}_{\mathrm{NAF}} \cdot \mathbf{x}+\mathbf{n}^{\mathrm{NAF}}
$$

where

$\mathbf{H}_{\mathrm{NAF}}$ is the channel coefficient matrix given by

$$
\mathbf{H}_{\mathbf{N A F}}=\left[\begin{array}{cc}
w_{s d} h_{s d} & 0 \\
w_{s r} v_{r d} \beta h_{s r} h_{r d} & w_{s d} h_{s d}
\end{array}\right]
$$

and

$$
\mathbf{n}^{\mathbf{N A F}}=\left[\begin{array}{c}
n_{s d} \\
v_{r d} h_{r d} \beta n_{s r}+n_{r d}
\end{array}\right]
$$

is the noise vector.

Assuming that the channel coefficient matrix $\mathbf{H}_{\mathrm{NAF}}$ is known or can be estimated, Maximum Likelihood (ML) decoding can be used at receiver to fully explore the diversity advantage of the scheme. The ML estimate of the transmitted packets is presented as follows: 


$$
\hat{\mathbf{x}}=\arg \min _{\mathbf{x}}\left\|\mathbf{n}^{\mathbf{N A F}}-H \mathbf{x}\right\|_{F}^{2}
$$

where $\|\cdot\|_{F}$ represents the Frobenius-2 norm, $H$ represents the estimated channel coefficient matrix and $\mathrm{x}$ takes all possible finite values depending on the signal constellation.

\subsection{Non-Orthogonal protocol with DF strategy (NDF)}

In DF strategy, the signal received at the destination and relay during the first time slot is identical to that for the AF strategy. However, the relay now should demodulate and decode the signal received during the first time slot. Assuming that the signal is decoded correctly and retransmitted, and we obtain

$$
y_{2}^{N D F}=w_{s d} h_{s d} x_{2}+v_{r d} h_{r d} y_{s r}+n_{r d}
$$

the transmission function of the DF strategy is

$$
\mathbf{y}^{\mathrm{NDF}}=\mathbf{H}_{\mathrm{NDF}} \cdot \mathbf{x}+\mathbf{n}^{\mathrm{NDF}}
$$

where

$$
\mathbf{H}_{\mathbf{N D F}}=\left[\begin{array}{cc}
w_{s d} h_{s d} & 0 \\
w_{s r} v_{r d} h_{r d} & w_{s d} h_{s d}
\end{array}\right]
$$

and

$$
\mathbf{n}^{\mathbf{N D F}}=\left[\begin{array}{c}
n_{s d} \\
n_{r d}
\end{array}\right]
$$

\subsection{Orthogonal protocols with the AF and DF strategies (OAF and ODF)}

In the orthogonal protocol with the AF strategy, the received signal can be written as

$$
\mathbf{y}_{\mathbf{2}}^{\mathbf{O A F}}=\left[\begin{array}{c}
w_{s d} h_{s d} \\
w_{s r} v_{r d} \beta h_{s r} h_{r d}
\end{array}\right] \mathbf{x}+\mathbf{n}^{\mathbf{O A F}}
$$

where $\mathbf{n}^{\mathbf{O A F}}$ is the $2 \times 1$ vector which is scalar $\mathcal{C N}\left(0, N_{0}\right)$ additive noise.

Similarly, the signal received at the destination terminal under the orthogonal protocol with DF strategy is

$$
\mathbf{y}_{\mathbf{2}}^{\mathbf{O D F}}=\left[\begin{array}{c}
w_{s d} h_{s d} \\
w_{s r} v_{r d} h_{r d}
\end{array}\right] \mathbf{x}+\mathbf{n}^{\mathbf{O D F}} .
$$

\section{INFORMATION THEORETIC PERFORMANCE ANALYSIS}

The mutual information of the AF and DF strategies are

$$
I_{A F}=\frac{1}{2} \log _{2} \operatorname{det}\left(\mathbf{I}_{\mathbf{2}}+\frac{1}{N_{0}} \mathbf{H}_{\mathbf{A F}}{ }^{H} \mathbf{H}_{\mathbf{A F}}\right)
$$


and

$$
I_{D F}=\frac{1}{2} \log _{2} \operatorname{det}\left(\mathbf{I}_{\mathbf{2}}+\frac{1}{N_{0}} \mathbf{H}_{\mathbf{D F}}{ }^{H} \mathbf{H}_{\mathbf{D F}}\right),
$$

respectively, where the superscripts ${ }^{H}$ stand for conjugate transposition; $\mathbf{I}_{2}$ represents $2 \times 2$ identity matrix; and $\mathrm{H}_{\mathrm{AF}}$ and $\mathrm{H}_{\mathrm{DF}}$ mean the channel matrix of $\mathrm{AF}$ and DF strategies.

Note that $I_{N A F}=I_{N A F}\left(\mathbf{y}_{2}^{\mathbf{N A F}} ; \mathbf{x}\right)$, where $I_{N A F}\left(\mathbf{y}_{\mathbf{2}}^{\mathbf{N A F}} ; \mathbf{x}\right)$ is the mutual information between the vectors $\mathrm{y}_{2}^{\mathrm{NAF}}$ and $\mathrm{x}$. Using the chain rule for mutual information, we have

$$
I_{N A F}=I_{N A F}\left(\mathbf{y}_{\mathbf{2}}^{\mathbf{N A F}} ; x_{1}\right)+I_{N A F}\left(\mathbf{y}_{\mathbf{2}}^{\mathbf{N A F}} ; x_{1} \mid x_{2}\right),
$$

where $I\left(\mathbf{y}_{\mathbf{2}}^{\mathbf{N A F}} ; x_{1}\right)$ is the mutual information between $\mathbf{y}_{\mathbf{2}}^{\mathbf{N A F}}$ and $x_{1}$; the $I\left(\mathbf{y}_{\mathbf{2}}^{\mathbf{N A F}} ; x_{1} \mid x_{2}\right)$ is the conditional mutual information between $\mathbf{y}_{2}^{\mathrm{NAF}}$ and $x_{1}$ with a given $x_{2}$. It is easy to verify that $I_{N A F}\left(\mathbf{y}_{2}^{\mathbf{N A F}} ; x_{1} \mid x_{2}\right)=I_{O A F}$. Then

$$
I_{N A F}=I_{N A F}\left(\mathbf{y}_{\mathbf{2}}^{\mathbf{N A F}} ; x_{1}\right)+I_{O A F} .
$$

Since $I\left(\mathbf{y}_{\mathbf{2}}^{\mathbf{N A F}} ; x_{1}\right) \geq 0$, we have $I_{N A F} \geq I_{O A F}$. This shows that the achievable rate of NAF is higher than that of OAF.

Similarly, the mutual information for the NDF and ODF satisfies:

$$
I_{N D F} \geq I_{O D F}
$$

This shows that the achievable rate for non-orthogonal protocol is higher than that for orthogonal ones, since non-orthogonal protocol can realize a multiplexing gain.

We consider the outage probability [1][3] at transmission rate $R$ for the orthogonal and nonorthogonal protocols base on the mutual information. The definition or outage probability concerning the mutual information $I$ and transmission rate $R$ is

$$
O=P(I \leq R)
$$

And the diversity order $(D)$ for transmission rate $(R)$ can be defined as

$$
D=\lim _{S N R \rightarrow \infty} \frac{-\log P(I \leq R)}{\log S N R}
$$

In case of NAF, we obtain

$$
I_{N A F}=\frac{1}{2} \log _{2}\left(1+\left(1+\frac{1}{\bar{\omega}^{2}}\right) \frac{\omega_{s d}^{2} h_{s d}^{2}}{N_{0}}+\frac{\omega_{s}^{2} \omega_{r d}^{2} h_{s r}^{2}}{\bar{\omega}^{2}\left(\omega_{s r}^{2}+N_{0}\right) N_{0}}+\left(\frac{\omega_{s d}^{2} h_{s d}^{2}}{\bar{\omega} N_{0}}\right)^{2}\right),
$$


where $\bar{\omega}=\sqrt{1+\frac{\omega_{r d}^{2}}{\omega_{s r}^{2}+N_{0}}}$.

And then, we can give the upper-bound of the outage probability according to

$$
P\left(I_{N A F} \leq R\right) \leq P\left(h_{s d}^{2}+h_{s r}^{2} \leq \frac{2^{2 R}-1}{\phi^{N A F}}\right)
$$

where

$$
\phi^{N A F}=\min \left\{\left(1+\frac{1}{\bar{\omega}^{2}}\right) \frac{\omega_{s d}^{2} h_{s d}^{2}}{N_{0}}, \frac{\omega_{s r}^{2} \omega_{r d}^{2} h_{s r}^{2}}{\bar{\omega}^{2}\left(\omega_{s r}^{2}+N_{0}\right) N_{0}}\right\}
$$

For $\phi^{N A F}$ sufficiently large, we have

$$
P\left(I_{N A F} \leq R\right) \leq\left(\frac{2^{2 R}-1}{\phi^{N A F}}\right)^{2} .
$$

referring to the definition of $D$, it can achieve the second-order diversity in in effective SNR $\phi^{N A F}$.

Similarly, we have

$$
P\left(I_{O A F} \leq R\right) \leq\left(\frac{2^{2 R}-1}{\phi^{O A F}}\right)^{2}
$$

and by inspection we finally obtain the order of effective SNRs

$$
\phi^{N A F} \geq \phi^{O A F}
$$

We can see that both orthogonal and non-orthogonal protocols can achieve second-order diversity in their effective SNRs, and the result of (29) is owing to the array gain realized by the virtual multiple-antennas in non-orthogonal protocols.

\section{SPACE-TIME CODING IN NON-ORTHOGONAL PROTOCOL}

Space-time coding is a method employed to improve the reliability of data transmission in wireless systems by using multiple transmit antennas. It relies on redundant copies of a signal to the receiver in the hope that at least some of them may survive the physical path between transmission and reception. Space-time codes may be split into two main types: space-time trellis coding (STTC) [5] and space-time block coding (STBC) [6]. We are only concerned here with STBC which acts on a block of data at once (similarly to block coding) and provide only diversity gain, but are much less complex in implementation terms than STTC.

Alamouti coding [4] is typical example of STBC, which proposed a simple $2 \times 1 \mathrm{MIMO}$ scheme that achieves a full diversity gain [6] with a simple maximum likelihood (ML) decoding algorithm. The transmit symbols are modulated using an $\mathrm{M}$-ary modulation scheme, then the encoder takes a block of two modulated symbols $s_{1}$ and $s_{2}$ in each encoding operation and sends it to the transmit antennas according to the code matrix,

$$
C_{1}=\left[\begin{array}{ll}
x_{1} & x_{2} \\
x_{3} & x_{4}
\end{array}\right]=\left[\begin{array}{cc}
s_{1} & -s_{2}^{*} \\
s_{2} & s_{1}^{*}
\end{array}\right]
$$


where $*$ denotes complex conjugate. In this code matrix, the first column represents the first timeslot (transmission period) in MIMO system [11] and the second column represents the second time-slot. The first row corresponds to the symbols transmitted from the first antenna and the second row corresponds to the symbols transmitted from the second one. This implies that the symbols are transmitting both in space (across two antennas) and time (two transmission intervals), that is to say, space-time coding. The traditional Alamouti coding is designed for a two transmit antennas system. Consider the cooperation method using the single-relay AF channel as FIGURE 3 , we define $d_{1}=\left(x_{1}, x_{2}\right)$ and $d_{2}=\left(x_{3}, x_{4}\right)$. Thus, in the first time-slot, the source sends $d_{1}$ to relay and destination; and in the second time-slot, the source sends $d_{2}$ and relay sends symbols received at first time-slot $d_{1}^{\prime}$ to destination, respectively. Then the Alamouti coding cooperative transmission function can be written as,

$$
\begin{gathered}
{\left[\begin{array}{ll}
y_{1} & y_{2} \\
y_{3} & y_{4}
\end{array}\right]=\left[\begin{array}{cc}
w_{1} h_{s d} & 0 \\
\beta v_{2} w_{1} h_{s r} h_{r d} & w_{2} h_{s d}
\end{array}\right]\left[\begin{array}{ll}
x_{1} & x_{2} \\
x_{3} & x_{4}
\end{array}\right]+} \\
{\left[\begin{array}{cc}
z_{s d 1} & z_{s d 2} \\
\beta v_{2} h_{r d} z_{s r 1}+z_{r d 1}+z_{s d 3} & \beta v_{2} h_{r d} z_{s r 2}+z_{r d 2}+z_{s d 4}
\end{array}\right]}
\end{gathered}
$$

where $y_{i}(i \in\{1,2,3,4\})$ is the symbol received at destination $d ; w_{i}(i \in\{1,2\})$ is square root of the average signal energy received at destination during the two time slots; $v_{2}$ is square root of the average signal energy received at relay; $h_{s d}, h_{r d}$ and $h_{s r}$ are the random, complex valued and unit-power channel gains between $s \rightarrow d, r \rightarrow d$ and $s \rightarrow d$, respectively; $z_{s d i} \sim \mathcal{C N}\left(0, N_{s d i}\right)$ $(i \in\{1,2,3,4\}), z_{s r i} \sim \mathcal{C N}\left(0, N_{s r i}\right)(i \in\{1,2\})$ and $z_{r d i} \sim \mathcal{C N}\left(0, N_{r d i}\right)(i \in\{1,2\})$ are the additive noises.

\section{NUMERICAL RESULTS}

In this section, some simulation results were shown to illustrate the performance of the presented approach. The wireless channel was simulated according to the sum-of sinusoids simulation model for Rayleigh fading channels of [7], according to which, each channel multi-path is a zero mean complex Gaussian random variable. We consider the single-relay cooperation model, and assume there is the same distance between all the terminals. We performed an experiment consisting of 100,000 independent frames. The length of frame was fixed to $N=600$ bits. Each frame contained BPSK symbols. ML decoding was used at the receiver to fully explore the diversity advantage of the scheme.

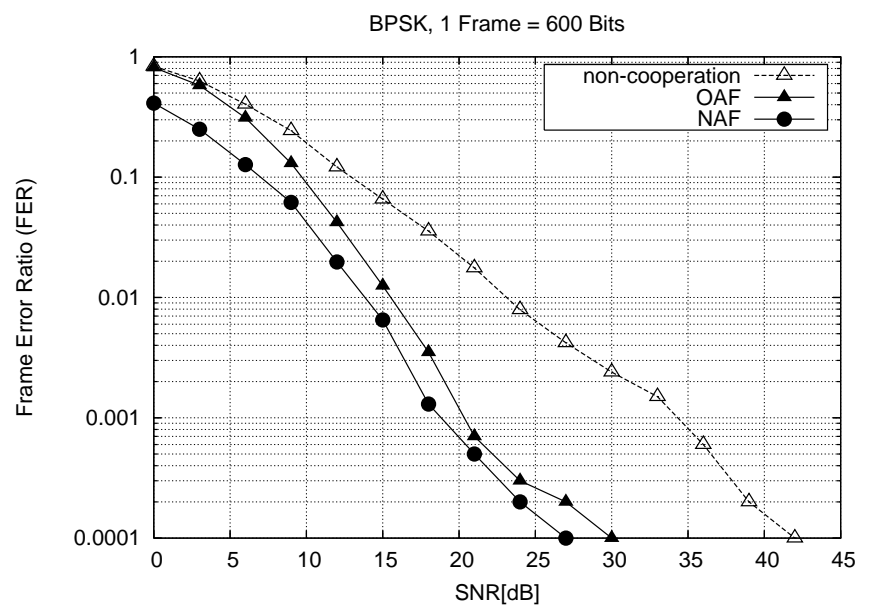

FIGURE 4: Non-cooperative, NAF and OAF cooperative protocols transmission by BPSK

Our simulation results are shown on FIGURE 4 and FIGURE 5 for the single-relay model of FIGURE 3. We find out that, the transmission frame error ratio performance of NAF cooperation 


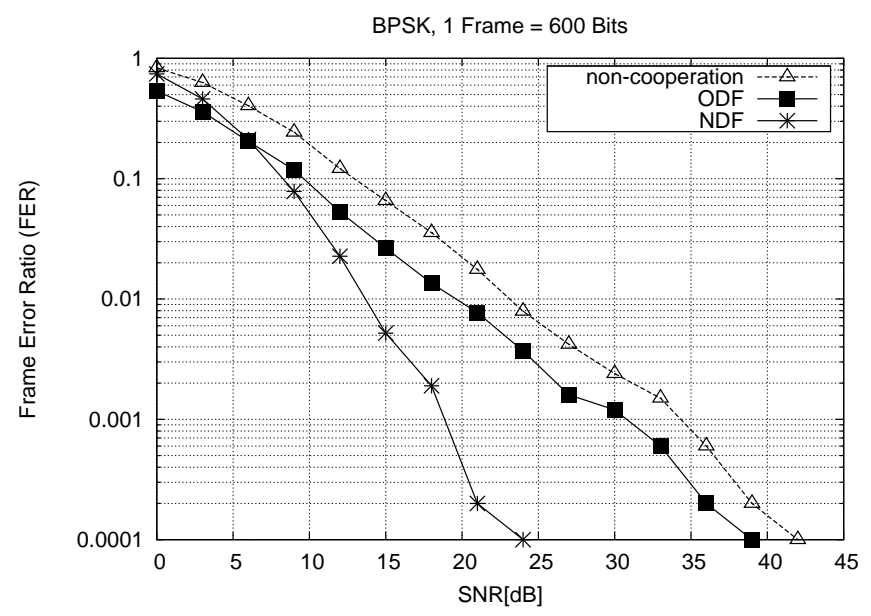

FIGURE 5: Non-cooperative, NDF and ODF cooperative protocols transmission by BPSK

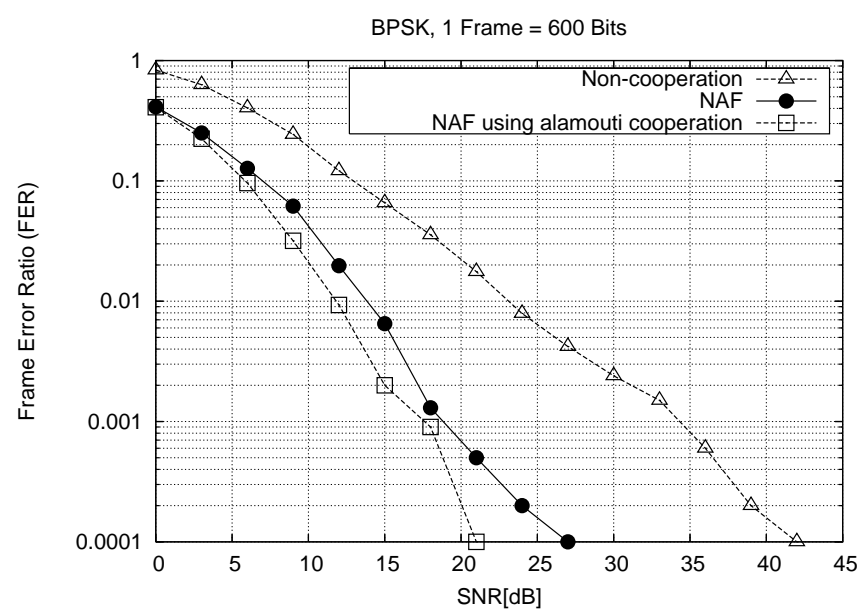

FIGURE 6: Non-cooperative, NAF and NAF using alamouti coding cooperative protocols transmission by BPSK

protocol outperforms both non-cooperative and OAF ones. Similarly, NDF has achieves a better performance than non-cooperative and ODF cooperation. The non-cooperation protocol transmit redundant symbols with the help of relay, so as to reduce the error ratio during the transmission.

In Alamouti coding cooperation, conjugate symbol was used to build up the quality of transmission. The simulation in FIGURE 6 is easy to show that with the space-time coding gains, it can achieve a better performance.

\section{CONCLUSION}

The focus of this paper is the performance of orthogonal and non-orthogonal cooperative protocols. Such a system is analyzed through a theoretical study and simulations to see the performance in a simple fading relay channel with AF and DF strategies. we analyzed the diversity performance of the different protocols through an outage probability analysis for each of the protocols. We show in this work that the non-orthogonal strategy offers optimal performance in the cooperative transmission. Furthermore, we introduce space-time coding in non-orthogonal cooperative protocols which provides the additional coding diversity gains of the transmission. 


\section{ACKNOWLEDGMENT}

This work comes within the framework of a project supported by the Agence Nationale de la Recherche/Réseau National de Recherche en Télécommunications under name RNRT/RADICSF/COMSIS and reference ANR-05-RNRT-014-01.

\section{REFERENCES}

[1] L. H. Ozarow, S. Shamai, and A. D. Wyner, "Information theoretic considerations for cellular mobile radio," IEEE Trans. Veh. Technol., vol. 43, no. 2, pp. 359-378, May 1994.

[2] L. Zheng and D. N. C. Tse, "Diversity and multiplexing: a fundamental tradeoff in multipleantenna channels," IEEE Transactions on Information Theory, vol. 49, no. 5, pp. 1073-1096, 2003.

[3] R. U. Nabar, H. Bolcskei, and F. W. Kneubuhler, "Fading relay channels: performance limits and space-time signal design", IEEE Journal on Selected Areas in Communications, Vol. 22, Issue 6, pp. 1099-1109, Aug. 2004.

[4] S. M. Alamouti, "A Simple Transmit Diversity Technique for Wireless Communications," IEEE Journal Select. Areas Commun., vol. 16, no. 8, pp. 1451-1458, Oct. 1998.

[5] V. Tarokh, N. Seshadri, and A. R. Calderbank, "Space-time codes for high data rate wireless communication: Performance analysis and code construction," IEEE Transactions on Information Theory, vol. 44, no. 2, pp. 744-765, Mar. 1998.

[6] V. Tarokh, H. Jafarkhani, and A. R. Calderbank, "Space-time block codes from orthogonal designs," IEEE Transactions on Information Theory, vol. 45, no. 5, pp. 1456-1467, Jul. 1999.

[7] Y. R. Zheng and C. Xiao, "Improved models for the generation of multiple uncorrelated Rayleigh fading waveforms," IEEE Commun. Lett., vol. 6, no. 6, Jun. 2002.

[8] J. N. Laneman and G. W. Wornell, "Energy-effcient antenna sharing and relaying for wireless networks," in Proc. IEEE Wireless Communcations and Networking Conference (WCNC), Chicago, IL, September 2000, pp. 7-12.

[9] J. N. Laneman, D. N. C. Tse, and G. W. Wornell, "Cooperative diversity in wireless networks: Effcient protocols and outage behavior," IEEE Transactions on Information Theory, Vol. 50, Issue: 12, pp. 3062-3080, Dec. 2004

[10] J. N. Laneman, G. W. Wornell, and D. N. C. Tse, "An effcient protocol for realizing cooperative diversity in wireless networks," in Proc. IEEE International Symposium on Information Theory (ISIT) ,Washington, D. C., June 2001, p. 294.

[11] J. G. Proakis, Digital Communications, Fourth edition. McGraw-Hill, 2001. 